\title{
Aspects of Orthogonality in the Development of the National Digital Wealth (NDW)
}

\author{
Ion IVAN ${ }^{1}$, Daniel MILODIN ${ }^{1}$, Sergiu CAPISIZU ${ }^{2}$ \\ ${ }^{1}$ Bucharest University of Economic Studies, Romania \\ ${ }^{2}$ Bucharest Bar Association \\ ionivan@ase.ro,daniel.milodin@ase.ro, capisizu@ew.ro
}

There are presented aspects of orthogonality in the development of the national digital wealth. There is presented the concept of NDW. Are identified quality characteristics. Are built orthogonality metrics for software development applications which are parts of NDW.

Keywords: National Digital Wealth, Metrics, Orthogonality

$\mathbf{1}_{\mathrm{D}}^{\mathrm{N}}$

National Digital Wealth (NDW)

Digital national wealth is a concept that has emerged with the first electronic computing machines and with the first electronic products developed with them. Emergence of electronic computing machines has led to the development of software products machine code and to the creation of files. The development of hardware and software components led to increased default spectrum applicability of the concept of NDW.

NDW concept involves finding solutions for creating software for the NDW development, NDW storage required, and involves the construction of means used to access NDW.

NDW is comprised of software, databases, multimedia content, resulted from the digitization process as celluloid film digital, music disc - $\mathrm{CD}$, e-learning platforms.

NDW components are:

- hardware: computers, routers, switches, fiber optic cables, data acquisition equipment - scanner, keyboard, digital camera, microphone, building design tools;

- software, which refers to computer applications, software products, along with related files and databases.

Unlike other components, NDW software component is the result of intellectual activity.

NDW is classified based on several criteria, taking into account the homogenization of the components resulted when applying the classification criteria.
By the criterion of components used NDW:

- products used directly to obtain information, services, transactions, results, training of the users;

- components that assist software development processes, industries producing consumer goods, industry that produces inputs for the production of consumer goods..

By the criterion of using mode, the NDW components NDW are:

- produced on a computer, working independently, without interaction with other components and without the need to interconnect with other components;

- networking products, network software that can be updated, the update should take into account components compatible versions;

- products in which software is on the network server and the database is also in the network..

After data entry criterion, the components NDW are:

- products which are activated and have no dynamic in terms of taking on new data; are independent components that do not interact with the user;

- products that take data, but do not store them, only use them when making selections; for example, the Google search engine takes data search results and subsequently identifies the option to perform new searches;

- products which allow string insert, allocate resources, select, pay and return 
it.

After typology of problems criterion NDW are games, sensors, console applications, elearning, e-peinture applications, applications for banking management, applications for achieving reservations, online projects homes, ERP software products, statistical processing, records population, migrations of people (passports), surveillance traffic, fingerprint, facial recognition, intelligent robots, image processing, audio processing, digitizing the tapes.

Based on complexity criterion, NDW components are:

- simple software applications, entering data and results are obtained for personal use;

- simple online programs that perform calculations on-line, for example determining BMI index for body weight;

- moderately complex programs in which data is entered, select the desired operations, are processed and low level access operation;

- complex programs;

- complex programs that require processing, allocating of resources, training, calculations, payments.

Based on quality criterion, NDW components are:

- untested programs with risks whose identification is required in order to reduce or eliminate them;

- partially tested programs;

- major tested programs, which remain in a proportion of $10-20 \%$ untested;

- fully tested programs, class A, found in nuclear power plants, aircraft, testing them is imperative because should not present any operational risk.

Based on availability criterion, NDW components are:

- online applications that are processed on the spot and make decisions, their role is to process data in real time;

- local applications that do not allocate resources.

Based on protection criterion, NDW components are:
- applications that are accessed under protection procedures;

- applications that are accessed freely without having to run over some steps by the user to be authenticated and access rights ascribed.

Based on time criterion, NDW components are:

- applications with real time processing, used to coordinate planes, chemical, nuclear power plant;

- application without real time processing used for payroll, tax amount establishment applications that simulate structural changes within communities.

NDW is an important component of national wealth, which requires a continuous growth and improvement. Ways to enhance digital content NDW involves reorganization, mitigation and control methods of destruction or damage to components NDW, improve the means of storage, archiving and processing, and the development of new types of support needed NDW storage.

Increasing NDW is achieved by :

- development of new manufacturing processes to achieve new generation products to ensure consistency between hardware, software, administrator and user requirements;

- establishing rigorous procedures for managing digital content versions in order not to loss by deletion, deterioration, updating or converting content structure;

- preservation software, databases, multimedia files and all other components of NDW in use, so as to keep the functionality for which it was created;

- introduction of computer security components at the organization's informatics system, as building different levels of security aims to produce contamination including components with high level of security which, in contact with unsecured component affected by on line attacks, produce negative and uncontrolled consequences on the entire system; 
- creating programs that perform data acquisition leading to conversions archives of documents, movies, music, book depots and magazines to digital format, which provides access to a growing number of people in the digital content specialized ;

- introduction of restrictions on the use of existing digital content, knowing that the use of copy-paste command does not create national wealth; restrictions are meant to cause all those who develop applications to bring new contributions, so that each component encompassing the added value, only in this way obtaining significant contributions NDW;

- use of quality metrics for digital content that will lead to the timing when a software product is considered part of the national wealth, because not every file or any database, any multimedia component, any site or any other web product is automatically an NDW component ;

- providing a level of redundancy control, because the takeover by copying parts of existing components in NDW enable versioning, it is important that every developer should take into account the dynamics of its products, so to be in circulation the newest version, which should contain all the facilities required by the previous versions.

NDW is a component formed by integrating the entire national wealth which exists in the online environment. Accessing them is done by custom module which allows viewing content rendered through a system connected online. So if the online environment requires adherence to necessary preparatory steps authentication and attribution of rights to view content stored, then NDW enforce procedures; if, however, is not required any authenticate and access is freely then NDW also allows free access to information.

\section{NDW Quality Features}

Digital national wealth is a concept with a broad spectrum of application, which requires knowledge of the quality characteristics in order to ensure the optimization of NDW components.

NDW quality features are given by the:

- reliability, hardware and software, is regarded as a measure of the ability of a NDW component to function properly in the conditions envisaged from the beginning. There are quantitative approaches of reliability, expressed as the probability that a component to perform certain functions and error-free performance in a time and in a given operating conditions, and qualitative approaches regarding component reliability as a NDW component capacity;

- portability, hardware and software, is defined as a set of attributes based on the facility that NDW components must have in order to be transferred from one medium to another. Environmental context means hardware and / or software and the organizational frame. NDW components are not considered portable not only if they are implemented on several computers directly, without change, but also whether the implementation on other types of computer systems requires small-scale changes and less effort than that required for modification of components for compatibility;

- NDW unique components involves issues as: each new component created to be different from the information already stored, thus seeking originality increase NDW components; when saving components within NDW should be considered and the situations where these components are stored several times;

- relevant show the components ability to express significant information to the user;

- orthogonality studying the originality of components and establishes the degree of resemblance between two or more components. 
A very important NDW characteristic is availability which is used to design the ways to work with NDW:

- the totally free accessibility is the characteristic of those informatics applications, equipment, facilitating the access to resources of informatics applications and consist in elimination of any restrictions, so anyone who sits at a terminal that is connected to the Internet, without special identification measurements to be able to access NDW components; through this access level users is able to dispose of all is stored in NDW;

- the partially restricted accessibility requires both functions that are freely accessible, without username and password, but also functions for which the user create an account, define a username and password and set those elements which allow him to perform processing; in the case of some applications where users are selected, they receive username, password and after that they access the application;

- the restricted accessibility is the feature of informatics applications that address user groups oriented on types of processing involving resources allocation with making payments or additions, alterations, respectively, removal of information; these informatics applications require the rigorous identification of users since there are responsibilities related to the nature of transactions by generated effects; in e-learning systems the tests require strict assessment on student; in electronic payments systems there is identified the account of the payee as well as the account of beneficiary that receives the amounts;

- the certified accessibility is a new type to obtain resources or to provide resources in the informational plan, the authentication being performed by electronic signature; the way how the electronic signature is defined gives the same value as a handwritten signature and even more.

Indicators are indicative, dynamic and by their aggregation result an indicator that offers a true picture of NDW.

\section{NDW Characteristics of Orthogonality}

The concept of orthogonality comes from mathematics.

Orthogonality is the indicator that studies the originality of an application based on the criteria of originality. The concept applies to software components, hardware and all data involved in the process of adding to the NDW.

Orthogonality establish the measurement in which the NDW components are taken or are specially designed to meet a need established, the hardware components perform functions uniquely, without any two or more hardware components to perform the same or similar activities and how data, information, images, tends to be redundant.

Orthogonality identifies those NDW components which must be revised to increase its efficiency, on the basis of orthogonality criteria.

Orthogonality indicator is calculated for each criterion, and the results are aggregated using the aggregation formula, resulting HA indicator, given by:

$$
H A=\sqrt[n]{\prod_{i=1}^{n} H_{i}}
$$

where:

$\mathrm{n}$ - the number of criteria used to analyze the NDW orthogonality;

$\mathrm{H}_{\mathrm{i}}$ - the value of $i$ orthogonality indicator.

Orthogonality criteria are dependent on the components evaluated and set standards for review of NDW.

Thus, if after determining orthogonality indicator of the component to be included, it has a value below 0.93 , its original level is one below the accepted limit and it is recommended not to be included.

Orthogonality NDW studying the degree of similarity between two or more components. Through this quality characteristics is determined if NDW meets the criteria of 
originality, both the components elements and at a unit level.

The concept of orthogonality comes from mathematics, where is considering the following aspects:

- two planes are orthogonal if their intersection angle cosine is equal to zero, a finite set of plane is orthogonal if planes are perpendicular in pairs;

- two lines are orthogonal if they form congruent adjacent angles, a finite set of lines is orthogonal if the lines are perpendicular in pairs;

- two vectors are orthogonal if their dot product is zero, a finite set of vectors is orthogonal if the dot product of any two different vectors is null.

Orthogonality is studied on the basis of orthogonality criteria. With these criteria are highlighted features that have the same value for the studied components and are determined levels of similarity. Using the concept of orthogonality is determined degree of similarity in the components already included in the NDW and between the new components to be included, in relation to the items already stored.

Based on the characteristics of the components there are defined their quality indicators. Based on these indicators is constructed an indicator that takes into account the values of component indicators.

Comparison of two components of NDW is reduced to report a component to the other, namely to identify common parts and parts that differ. There are then compared the corresponding features of the two components.

To study the orthogonality is defined a standardized indicator orthogonality in the range $[0,1]$, which takes the value 1 if the elements are orthogonal, that have nothing in common and the value 0 if the elements are identical, they have no different values for no feature.

If the indicator tends to 1 means that the components of NDW tend to orthogonality, and if the indicator is close to 0 means that the sets of components have many identical elements.
Study of NDW components orthogonality implies orthogonality import definitions and constructing mathematical indicators to be applied on NDW components .

Adapting mathematical formulas imposes studying the similarities between concepts and building indicators that satisfy the demands of working with NDW components. We propose the following algorithm for determining the orthogonality of the two NDW components.

In order to compare the new components to be taken in the NDW existing components, should be considered to define the classes of characteristics. Classes of characteristics the qualities of all components included in the NDW, and the quality of future components. Based on the classes of characteristics is provided a new filter for the newest elements, namely originality that new components bring to the NDW.

In order to be tested the originality, should be considered an important step, which is to determine which class of characteristics it belongs to the new component.

After determining the class of characteristics, there are compared all components already included in NDW with de new component, based on belonging to the same class 's characteristics; for each comparison are taken into account those characteristics for which the two compared terms have the same values. After testing all the components related to new component, the indicator is the aggregated, and if the value is above the threshold of $75 \%$, it is considered that the new component does not make any original contribution within NDW, otherwise the component is taken in the NDW .

There are presented the algorithm $\mathrm{P}_{\mathrm{i}}$ steps:

$\mathbf{P}_{\mathbf{1}}$ there are considered the quality characteristics $\mathrm{K}_{1}, \mathrm{~K}_{2}, \ldots, \mathrm{K}_{\mathrm{m}}$;

$\mathbf{P}_{\mathbf{2}}$ each characteristics class is composed by the characteristics: $\mathrm{K}_{1}=\left\{\mathrm{k}_{11}, \mathrm{k}_{12}, \ldots, \mathrm{k}_{1 \mathrm{n}}\right\}$, $\mathrm{K}_{2}=\left\{\mathrm{k}_{21}, \mathrm{k}_{22}, \ldots, \mathrm{k}_{2 \mathrm{n}}\right\}, \ldots, \mathrm{K}_{\mathrm{m}}=\left\{\mathrm{k}_{\mathrm{m} 1}, \mathrm{k}_{\mathrm{m} 2}\right.$, $\left.\ldots, \mathrm{k}_{\mathrm{mn}}\right\}$;

$\mathbf{P}_{\mathbf{3}}$ each component already included in NDW is given to a class of characteristics; 
$\mathbf{P}_{\mathbf{4}}$ it is considered the $\mathrm{T}$ digital component which is tested in order to be established if it will be included in NDW;

$\mathbf{P}_{5}$ it is identified the characteristics class to which the T component belongs to;

$\mathbf{P}_{6}$ for all the components already included in NDW and which belongs to the same class of characteristics with the new component, it is calculated the aggregated indicator:

where:

$$
\mathrm{H}_{\mathrm{k}}\left(\mathrm{T}_{\mathrm{j}}, \mathrm{T}\right)=\frac{\sum_{i=1}^{m} I\left(K_{j i}, K_{T i}\right)}{E\left(K_{T}, K_{j}\right)},
$$

$\mathrm{T}_{\mathrm{j}}$ - the $i$ already included in NDW

$\mathrm{T}$ - the component to be included in NDW;

$\mathrm{m}$ - the number of the characteristics classes;

$\mathrm{K}_{\mathrm{ji}}$ - the characteristic for component $i$ for the $j$ class of characteristics;

$\mathrm{E}()$ - the number of characteristics of the class for which the new component and the component already included have similar values.

For component to be included it is identified a class of characteristics $K_{T}$ which is the same as the characteristics class for the component already included and in order to establish the degree of originality, compared to NDW components, are aggregates $\mathrm{H}_{k}$ indicators.

The components' orthogonality is determined as internal and external. Internal orthogonality sets the extent to which the component elements are used repeatedly.

External orthogonality shows the differences and similarities that exist between components.

Building orthogonality indicator provides a touch of originality to the items included in the NDW. Storage elements that bring new utility helps to increase NDW, users becoming more interested in share the NDW components.

Independent developers develop simultaneously NDW components with the same functionality, aspect which involves the development of the indicators to ensure the inclusion of the original components in the NDW.
In order to establish the components' degree of originality was developed the on-line ORTOES application. This allows the calculation of the orthogonality level for a $\mathrm{C}++$ class of components, composed of 130 components.

The ORTOES application presents the characteristics needed to be extended in order to use them to determine the originality of components NDW.

\section{NDW Orthogonality Metrics}

Software metric is a mathematical model that contains one or more equations or inequalities, and has one or more objective functions; its role is being to describe the associated system status. Metrics are models implemented to test the quality of NDW components, taking into account factors influencing the measured characteristic.

The need to use metrics is given by the following considerations:

- Allow setting targets for improvement NDW components;

- Provides a real way to achieve these goals;

- Allow the identification of the causes that negatively affect the characteristics of NDW quality components;

- Identify requirements to be followed for the development of component models qualitatively superior.

The metric is a definition, an algorithm or a mathematical function used for quantitative evaluation of the product tested.

A metric is designed to achieve the following objectives:

- Quantification of characteristics;

- Determine the influence of indirect factors;

- Aggregation of values;

- Hierarchy of components;

- Comparative analysis.

There are presented the metric's functions:

Measurement function, with which is emphasized the quality characteristics measurement level, by expressing the metric units into the measurement units.

Comparison function, define the purpose of using the quality metrics, namely to analyze 
from the point of view of a quality features parts of NDW and to make comparisons with herself, fitting into a defined category of components, or with other components placing them at a certain stage in the hierarchy. Comparison is limited to the achievement gap between the two terms expressed in the same unit of measure comparing the result to 0 , or calculating the ratio, with verifying the result to 1 .

Analysis function gives meaning to the numerical results obtained by applying mathematical models associated to the metrics. Based on numbers obtained, NDW components are given quality characteristics. Function analysis helps to generate a lot of homogeneous components by finding and removing individual cases or excessive.

Synthesis function is used when investigating NDW groups components and components are grouped in homogeneous lots, and based on this lots are synthesized the essential characteristics for all NDW.

Estimation function, through which the software metric is used to measure the trend of increase / decrease in NDW quality components.

Test function, shows that the results obtained applying the software metrics are used to confirm and strengthen or refute the conclusion obtained through other methods. The use of software metrics in practice involves hypothesis validation and independence of results; for complex characteristics, whose study requires aggregate elements, verification is done by decomposing each primary component features and analyze them separately.

The metrics of NDW digital component are influenced by:

- Computing capacity

- Internet access capability

- Number of users

- The amount of software

- Volume database

- Indicators of complexity: software, databases, digital content, national wealth.

This approach introduces:

- Approximation of the failure to include components;

- Selective given by the model used;

- Subjective approach by prioritizing important criteria obtained from a collectivity formed by experts, even if it is representative, this is not shown.

The NDW amount and structure are important for:

- Allocation of funds to purchase equipment;

- Define strategies for future development. NDW study must be approached with the idea of establishing the way forward for increasing the equipment modernization and for increasing skill levels.

Implementation and a continuous analysis of NDW increase culture degree, access to information, automation of daily activities and a thorough knowledge of the qualitative aspects of life. NDW data components have the first criterion storage the high levels of accuracy and a redundancy as low as possible.

The $D N W$ indicator is estimated starting from the formula:

$D N W=\sum_{i=1}^{n h w} p 1_{i} * H W_{i}+\sum_{j=1}^{n s w} p 2_{j} * S W_{j}$

where:

$H W_{i}$ - the value of $i$ hardware component;

$S W_{j}$ - the value of $j$ software component;

$p 1_{i}, p 2_{j}$ - the weight of distinct hardware and software components;

$n h w$ - the number of hardware components;

$n s w$ - the number of software components.

NDW is a component for users, not for developers, it is a citizen oriented application, aspect involving:

- Quality characteristics must be constructed by adapting to user needs;

- Development cycles must starts from the analysis of the target groups to which NDW is addressed, following that, with the completion of basic cycles, to consider extending the general public;

- Development structure for NDW must be built taking into account the maximization of user satisfaction 
If it is taking into consideration the level of satisfaction for users who accessed NDW, it is determined by applying the formula:

$$
N S U=\frac{N U F}{T N U}
$$

where:

$N U F$ - the number of users which accessed NDW and identified that NDW component which they were looking for, component which once founded doesn't imply new search;

$T N U$ - the total number of users which accessed NDW and identified or didn't identify what they were looking for.

It is established the relative satisfaction indicator, RSL:

$$
R S L=\frac{N U T}{T N U}
$$

where:

$N U T$ - the number of users which repeatedly accessed NDW and which after many attempts identified what they were looking for;

$T N U$ - total number of users.

RLS is taking value between 0 and 1 , the 0 limit showing a small degree of satisfaction.

NDW is an open system, which is based on the concept of inclusion, through is taken new components which are the subject of national wealth in digital form. Acquisition of new components is realized by analyzing their content, as follows:

- Identifying the content already stored in NDW;

- Strict control of redundancy to provide original content and a high utility to the user;

- Analyzing and retrieving components containing complex content;

- Implementing processes linking content already stored with new content of the taken components.

Determining metrics must meet the theoretical, conceptual described in mathematics and statistics. Primary indicators are those that are obtained by numerical quantification based on considered quality characteristics.

Metrics are the way by which numerical form elements that define the quality of the components included in the NDW are translated into results. Implementation of the metrics involves identifying quality characteristics, influencing factors, establishing links between factors of influence, building primary indicators and interpretation of results.

\section{Conclusions}

NDW is a complex process that involves analyzing, processing and storage of components which are accessed through an electronic calculating system. The NDW components range from software, hardware and continue with all areas in which they are applicable and useful.

Homogenization the NDW content is one aspect that should be considered in identifying, analyzing and incorporating new components.

The spread of NDW is an accurate indicator of the level of automation of the company, the degree of socialization of the community members and especially it is an accurate measure of the level of culture and development of the society.

NDW evolution involves the acquisition of data so as to enable increasing the utility for the users. In order to include original data, the orthogonality indicators which are testing the components quality should be improved, their range of applicability must be extended so as to improve the quality of NDW components.

Building digital wealth leads to a new digital component, namely the knowledge society.

\section{References}

[1] I. Ivan, C. Ciurea, Gh. Nosca, "Considerations About d-National Wealth", Proceedings of the 12th International Conference on Informatics in Economy, IE 2013, April 25-28, 2013, Bucharest, Romania, ASE Printing House, ISSN 2284-7472.

[2] I. Ivan, C. Ciurea, A. Zamfiroiu, Gh. Noșca, "Characteristics of d-National Wealth in the Knowledge-based Economy", Economy Informatics, vol. 13, no. 1-4, 2013, pp.51-63 
[3] M. Manescu, "National wealth", RSR Academy Printing House, Bucharest, 324 pg.

[4] A. Smith, "Wealth of nations", ALL Printing House, Bucharest, 2010, 96 pg, ISBN 978-973-5711-976-0

[5] I. Ivan, D. Milodin, S. N. Dumitru, "C++ programs orthogonality software mining", Romanian Journal of Information Technology and Automatic Control, vol. 17, no. 2, 2007, pg. $39-54$

[6] D. Milodin, L. Săcuiu, "Orthogonality problems for non-homogenous informatics applications", Young Researcher International Symposium, (VIth edition) ASEM Chisinau, 18 - 19 of April, 2008, vol. I, ASEM Printing
House, Chisinau, 2008, pg. 341-343.

[7] D. Milodin, S. Dumitru, "C++ programs' orthogonality in software mining, Informatics in knowledge society", The proceedings of the eight international conference on informatics in economy, ASE Printing House, Bucharest, 2007, pg. $913-918$

[8] I. Ivan, C. Ciurea, D. Milodin, "Collaborative Educational System Analysis and Assessment", Proceedings of The Third International Conferences on Advances in Computer-Human Interactions, ACHI 2010, February 1016, 2010, Saint Maarten, Netherlands Antilles, ISBN 978-0-7695-3957-7

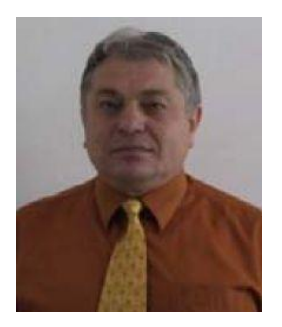

Ion IVAN has graduated the Faculty of Economic Computation and Economic Cybernetics in 1970. He holds a PhD diploma in Economics from 1978 and he had gone through all didactic positions since 1970 when he joined the staff of the Bucharest Academy of Economic Studies, teaching assistant in 1970, senior lecturer in 1978, assistant professor in 1991 and full professor in 1993. Currently he is full Professor of Economic Informatics within the Department of Computer Science in Economics at Faculty of Cybernetics, Statistics and Economic Informatics from the Academy of Economic Studies. He is the author of more than 25 books and over 75 journal articles in the field of software quality management, software metrics and informatics audit. His work focuses on the analysis of quality of software applications. He has participated in the scientific committee of more than 20 Conferences on Informatics and he has coordinated the appearance of 3 proceedings volumes for International Conferences. From 1994 he is $\mathrm{PhD}$ coordinator in the field of Economic Informatics. His main interest fields are: software metrics, optimization of informatics applications, developments and assessment of the text entities, efficiency implementation analysis of the ethical codes in informatics field, software quality management and data quality management.

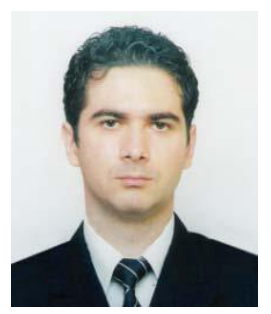

Daniel MILODIN graduated the Economic Informatics department of the Faculty of Economic Cybernetics, Statistics and Informatics in 2005. He followed the master program Project's Computerized Management between 2005 and 2007. He has published several articles in specialized magazines, articles focused mainly on studying and implementing the concept of orthogonality: the orthogonality of the alphabets, of the Arabic digits, of the Latin alphabet, of the distributed informatics applications and of the structured entities. The dissertation paper within the master is placed on the same line of research, "The projects' orthogonality, conditions of entry into the assessment program", proposing to define the projects' concept of orthogonality, methods for determining the degree of similarity between two projects, and also to develop software products for identifying similar projects. He holds a PhD diploma in Economics, specialization "Economic Science", with the theme "The orthogonality of the structured entities". 


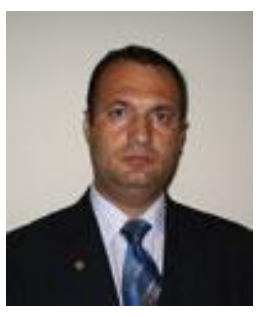

Sergiu CAPISIZU has graduated the Faculty of Cybernetics, Statistics and Economic Informatics in 1997 and National University of Defense in 2005. He holds a PhD diploma in Economic Cybernetics and Statistics, having the title Models and techniques to perform the economic information audit. He is co-author of books and articles in information audit and ICT fields. Also, he has published articles in proceedings of national and international conferences, symposiums, workshops in the fields of data quality, software quality, information audit and juridical aspects in ICT field. He is evaluator of ANEVAR association. 